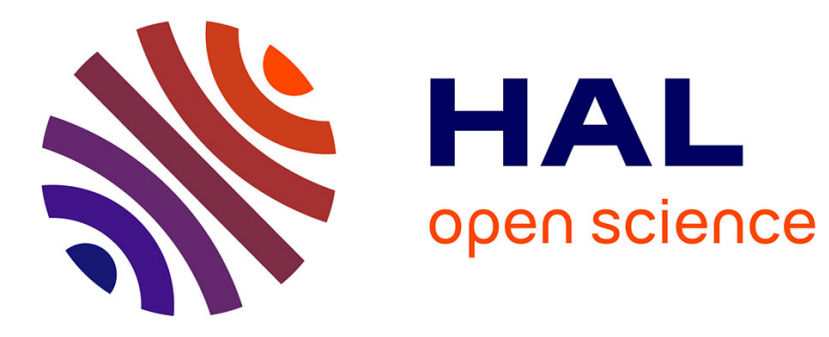

\title{
Unexpected phenotype in a patient with two chromosomal deletions involving 6pter and 22q11
}

Q. Salardaine, V. Marquet, S. Bourthoumieu, B. Dauriat, A. Dobrescu, C. Yardin

\section{- To cite this version:}

Q. Salardaine, V. Marquet, S. Bourthoumieu, B. Dauriat, A. Dobrescu, et al.. Unexpected phenotype in a patient with two chromosomal deletions involving 6pter and 22q11. Morphologie, 2019, 103, pp.116 - 121. 10.1016/j.morpho.2019.02.002 . hal-03484650

\section{HAL Id: hal-03484650 \\ https://hal.science/hal-03484650}

Submitted on 20 Dec 2021

HAL is a multi-disciplinary open access archive for the deposit and dissemination of scientific research documents, whether they are published or not. The documents may come from teaching and research institutions in France or abroad, or from public or private research centers.
L'archive ouverte pluridisciplinaire HAL, est destinée au dépôt et à la diffusion de documents scientifiques de niveau recherche, publiés ou non, émanant des établissements d'enseignement et de recherche français ou étrangers, des laboratoires publics ou privés.

\section{(ㄷ)(1) $\$$}

Distributed under a Creative Commons Attribution - NonCommercial| 4.0 International 


\section{Médecine et Droit}

\section{Protection de la personne}

Fin de vie ... encore et toujours -

End of life... again and again

Aline Cheynet de Beaupré

Aline.cheynet-de-beaupre@univ-orleans.fr

Professeur de Droit privé à l’Université d’Orléans

12 bis, rue du Temple - 21000 DIJON

$0614180377-$

\section{Résumé}

La fin de vie n'est pas la question au cœur des Etats généraux de révision des lois de bioéthique françaises - et n'a pas à l'être- , mais l'étude réalisée par le Conseil d'Etat en juin 2018 se présente comme un modèle du genre qui gagnerait à devenir le rapport de référence sur la question de la fin de vie lorsqu'il s'agira, un jour peut-être de la réviser.

Mots clés: Fin de vie; Soins palliatifs; Lois de Bioéthique

\section{Abstract}

End of life is not the question at the heart of the Estates General purporting to the revision of the French bioethics laws - and it does not have to be -. However, the study carried out by the Council of State in June 2018 presents

itself as a model of its kind, that should be entitled to become the reference report on the end-of-life issue, when the time will come, one day perhaps, to revise it.

Key Words: End of life; Bioethics laws; Palliative care

« L'erreur fondamentale de nos pensées binaires est d'opposer la mort à la vie. La vraie paire $d^{\prime}$ 'antonymes est naissance et mort, le passage du commencement et le passage de la fin. Et ce qui passe par ces deux portes et qui s'y engouffre, c'est, dans les deux cas, la vie. Ontologiquement la 
mort est comme la naissance, inhérente à la vie - et non son opposé $"{ }^{1}$.Juristes et non pas psychologues, les auteurs de l'Etude du Conseil d'Etat, réalisée en Juin $2018^{2}$, ont néanmoins présenté un travail académique presque irréprochable qui se nourrit non seulement des règles de Droit, mais également des contraintes et complexités humaines.

Leur Etude mériterait d'être dorénavant proposée comme texte de référence pour toute réflexion ultérieure relative à la question de la fin de vie. Point de passions, point de jugements, ni de leçons mais des prises de position et des clarifications présentées en connaissance de cause.

Les règles de droit existantes y sont analysées et pesées avec la même délicatesse qu'Osiris, le maître de la vérité, aurait pu jadis le faire sur la balance pesant l'âme du défunt pour sa justification.

Sur la fin de vie, les Etats généraux de la bioéthique ont refermé leur porte avec lassitude (I) autorisant quelques observations liminaires potentiellement irrespectueuses (II) avant que de laisser la place à une Etude du Conseil d'Etat qu'on aurait aimé voir placée plus haut sous le feu des projecteurs médiatiques (III).

I. Fin de vie : aperçu des débats régionaux des Etats généraux de la bioéthique La fin de vie n'était pas la reine de ces Etats généraux et ce, à plus d'un titre. Pourtant, il est intéressant d'en faire une analyse rapide pour identifier la place de la fin de vie au milieu des autres débats.

\section{1 Participation}

Concernant les rencontres régionales des Etats généraux, la représentation de la participation des citoyens sur la fin de vie a été proportionnellement beaucoup plus élevée que pour chacun des autres thèmes débattus pris séparément. Ce sujet précède l'AMP (assistance médicale à la procréation que le grand public désigne plus facilement sous l'acronyme PMA, procréation médicalement assistée) et le don d'organes qui sont, eux, parfois à équivalence de public. Les autres sujets (I'IA - Intelligence artificielle - ou les objets connectés constituant des sujets plus techniques et partant peut-être moins accessibles au grand public). En revanche, les statistiques de classement des " thèmes mobilisant le plus de citoyens par thème " replacent l'AMP, sans surprise en première position, suivie juste après par la fin de vie.

Plusieurs explications possibles à cela : la lassitude sur certains sujets (trop) médiatisés (PMA), la date dans le calendrier des révisions, un intérêt aigu... d'autres explications pouvant tenir au mode de diffusion des informations, la publicité, les lieux, les intervenants... Retenons néanmoins un engouement toujours fort pour le sujet de la fin de vie.

\footnotetext{
${ }^{1}$ Christiane Singer, Où cours-tu, ne sais-tu pas que le ciel est en toi ? Le livre de poche 2001, p. 126.

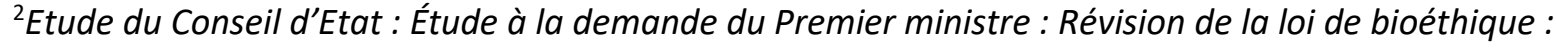
quelles options pour demain ? Étude adoptée en assemblée générale le 28 juin 2018, Conseil d'État, section du rapport et des études.
} 
Les professionnels de santé étaient fortement représentés, de façon constante, alors que les personnalités politiques étaient peu mobilisées. Sans surprise l'ADMD (Association pour le Droit à Mourir dans Dignité) répondait présente, mais également La Manif pour tous, qu'on aurait plutôt attendue dans les débats portant sur l'AMP...

- Quant aux directives anticipées :

Leur rédaction est toujours aussi difficile : seuls $5 \%$ des participants les avaient rédigées alors même que le public se mobilisant pour ces débats est un public sensibilisé à la question de la fin de vie et notamment aux directives anticipées...

A la question " Pourquoi ne pas avoir rédigé de directives anticipées ? ", le public présent (impliquant une relativité de la représentativité...) répond, s'excusant presque : n'être pas atteint d'une maladie chronique ou n'être pas issu du monde médical et rencontrer des difficultés de compréhension ou d'expression de ces directives. Des souhaits sont cependant exprimés manifestant notamment les attentes suivantes : il faudrait développer une relation de confiance avec les soignants, disposer de plus d'explications, bien exposer aux citoyens qu'il est possible de changer d'avis sur des directives précédemment exprimées.

Rien de nouveau pour les juristes (ni le monde médical) : ces questions, attentes ou regrets sont connus, ont été pris en considération dès 2005 puis à nouveau en 2016 lors de la révision de la loi sur la fin de vie. L'information issue de la loi de 2016, notamment, est mal relayée.

- Quant à l'euthanasie :

Les soignants soulignent (une nouvelle fois) une demande euthanasique très minoritaire des patients en fin de vie.

Pourtant, les participants ont voulu en parler. Les discussions portaient sur la liberté individuelle ou collective mais également sur l'auteur du geste à accomplir : soignant ou tiers ?

- Quant au suicide assisté :

Sur ce sujet « classique » en la matière, on note assez rapidement des confusions ou assimilations entre suicide et suicide assisté.

- Quant à la sédation profonde et continue :

Elle génère des inquiétudes du public sur de possibles dérives. Mais ce point mériterait d'être approfondi : quelles dérives ? Dérive légale ouvrant la voie à l'euthanasie ou dérive médicale de " libération de lits occupés ? ", entre autres questions...

- Quant à l'accompagnement des personnes en fin de vie :

Les participants pointent un manque d'accompagnement autour de la fin de vie distinguant la prise en charge médicale (bien notée) et l'accompagnement humain (à améliorer). Instaurer un temps d'écoute et de parole, « retisser » un lien social entre les différents intervenants... 
Ces observations, de l'ordre du psychologique ou du social, manifestent la difficulté du public à aborder le sujet de la fin de vie. Il ne s'agit pas de « bioéthique " à l'évidence et les lois sur la fin de vie ne sont pas en cause.

- Quant aux soins palliatifs :

Le développement des soins palliatifs est à nouveau sollicité malgré le Plan national triennal pour le développement des soins palliatifs et l'accompagnement en fin de vie - 2015-2018. Ce refrain, hélas récurrent, ne surprend pas et l'on notera, par ailleurs, que les soins palliatifs ne sont abordés dans les débats qu'en $3 e$ ou $4 \mathrm{e}$ lieu... Les propositions préconisent d'améliorer quantitativement et qualitativement la formation des soignants aux soins palliatifs d'une part, de valoriser et développer les soins palliatifs, d'autre part.

Les participants soulignent à juste titre que la « dignité » n'est pas un critère de définition de la fin de vie. De nombreuses personnes ont enfin exprimé la nécessité de laisser du temps à la loi et d'optimiser sa diffusion et sa connaissance.

\section{2 Bilan sommaire}

Le public présent (et donc sensibilisé) souligne de façon récurrente le manque d'information sur la connaissance de la loi sur la fin de vie, la sédation profonde et continue jusqu'au décès, l'euthanasie et le suicide assisté, ainsi que sur la rédaction des directives anticipées.

Sur la question de la potentielle légalisation de l'euthanasie et du suicide assisté les préconisations portent sur un encadrement très strict ; une clause de conscience pour les médecins; un corps de métier dédié à la pratique de l'euthanasie...

Les spécialistes de la question ne seront pas étonnés par ces constats, ni par l'une des conclusions : le manque d'informations des participants non soignants sur les termes de la fin de vie qui distinguent mal entre l'euthanasie, le suicide assisté et la sédation profonde et continue jusqu'au décès.

Discours déjà connus, constats et propositions attendus, attentes réitérées sur les soins palliatifs, mésinformation globale dénoncée mais que l'on serait tenté de présenter comme parfois volontaire. La mort est un tabou, ceux qui demandent qu'on en parle plus, écouteront-ils eux-mêmes plus ? Lebilan sur les directives anticipées si discutées, si sollicitées et ... si peu rédigées ne résume-t-il pas ces débats?

II. Prolégomènes et hors sujets

L'euthanasie avait-elle seulement sa place dans le débat sur la révision des lois bioéthiques ? Rien n'est moins sûr.

Diversion, démagogie, opportunisme de certains lobbies... la discussion pourrait être ouverte, mais ne connaîtrait vraisemblablement pas de solution ${ }^{3}$.

II 1 La fin de vie n'est pas une question de bioéthique ${ }^{3}$ V. not. : De l'opportunité d'une nouvelle loi sur la fin de vie ? (sous la dir. de L. Nicolas-Vullierme) (Elsevier,
Médecine § Droit, 14 juin 2018). 
Il est traditionnel de ne voir dans la " bioéthique " que les questions relatives aux biotechnologies susceptibles d'avoir des incidences éthiques. Les fécondations in vitro, la recherche sur les personnes vivantes, la génétique (" gène éthique »...), les dons d'organes, AMP, GPA (gestation pour autrui)...

Les précédentes lois et révisions de lois de bioéthique n'avaient pas traité de la fin de vie et leurs intitulés et objets en témoignent. La première loi, en 1994, était ainsi « relative au don et à l'utilisation des éléments et produits du corps humain, à l'assistance médicale à la procréation et au diagnostic prénatal ».

Pour réviser, encore faut-il avoir établi auparavant. Si établissement il y a eu, ce n'est pas en tant que loi de bioéthique. Le Conseil d'Etat lui-même, en 2009, relevait ce point ${ }^{4}$ et l'Agence de biomédecine dans son rapport de janvier 2018 ne mentionnait pas plus cette question ${ }^{5}$.

II 2 Loi Claeys/Leonetti : deux ans à peine !

$1994^{6}, 2004^{7}, 2011,2019$ : les lois de bioéthique ou de révision sont nombreuses et programmées.

$2005^{8}, 2016^{9}$ : spécifiques, les lois sur la fin de vie sont peu nombreuses, conjoncturelles et autonomes par rapport aux lois de bioéthique.

Mais surtout, la dernière loi en matière de fin de vie remonte à deux années à peine. La révision ne s'impose nullement, voire constituerait une atteinte à l'autorité et à la stabilité de la loi.

II 3 Rien de nouveau sous le soleil

Pour décourager le lecteur, on pourrait annoncer : que les débats des Etats généraux n'ont rien apporté et que l'Etude du Conseil d'Etat ne propose pas de révolution en la matière. Ce serait au risque de négliger une Etude de qualité permettant de présenter un état des questions précis juridiquement, juste humainement, et objectif.

Les lois sur la fin de vie de 2005 et 2016 révèlent un équilibre atteint humainement, médicalement et juridiquement. Sans surprise la nécessité du développement des soins palliatifs réapparaît.

Les sujets débattus trahissent peut-être la volonté et la peur de parler de la fin de vie. Il faut en parler. Mais c'est en public (dans l'anonymat et loin des soignants) que l'individu essaie de le faire et il ne rédige pas pour autant ses directives anticipées.

Les psychologues auront beaucoup à dire, le juriste se contentera de dire : Rien de nouveau sous le soleil. Les défenseurs des soins palliatifs (soignants et partisans) diront quant à eux : Quand auronsnous des crédits plutôt que des discussions?

${ }^{4} C E$, La révision des lois bioéthiques - Les Études du Conseil d'État, 6 mai 2009, p. 85.

${ }^{5} A B M$, Rapport sur l'application de la loi de bioéthique, janv. 2018.

${ }^{6}$ Loi ${ }^{\circ}$ 94-654 du 29 juillet 1994 relative au don et à l'utilisation des éléments et produits du corps humain, à I'assistance médicale à la procréation et au diagnostic prénatal.

${ }^{7}$ Loi n ${ }^{\circ} 2004-800$ du 6 août 2004 relative à la bioéthique.

${ }^{8}$ Loi n ${ }^{\circ}$ 2005-370 du 22 avril 2005 relative aux droits des malades et à la fin de vie.

${ }^{9}$ Loi n ${ }^{\circ}$ 2016-87 du 2 février 2016 créant de nouveaux droits en faveur des malades et des personnes en fin de vie. 
La conclusion de ces prolégomènes conduit à ne pas remettre en cause ce qui vient d'être voté et à regretter qu'on n'entende pas plus la voix du Conseil d'Etat, peut-être un peu étouffée par la médiatisation de l'avis du $\mathrm{CCNE}^{10}$ et le débat relatif à la « PMA pour toutes ».

III. Points cardinaux de l'Etude du Conseil d'Etat

L'Etude fut rendue en juin 2018, commencée bien auparavant, elle put néanmoins s'enrichir d'une partie des débats tenus auprès du grand public.

Pour le Conseil d'Etat, la question de la relation patient/médecin (il faudrait élargir à patient/soignant...) se résume en quatre points que sont : l'accès aux soins palliatifs ; le droit de refuser son traitement ; le refus de l'obstination déraisonnable ; l'interdiction (... pour le soignant) de provoquer la mort délibérément.

Le Conseil relève :

1. Qu'il faut assurer l'accès aux soins palliatifs : Cet accès aux soins passe par la question du développement des unités de soins palliatifs. Lapalisse aurait probablement souligné que pour y accéder, encore faudrait-il qu'il y en eût... Il faut savoir gré au Conseil d'avoir eu la lucidité (et le courage) de poser ce point à la première place. C'est manifester une compréhension en profondeur de la question de la fin de vie. Ce préalable, rappelé comme une litanie par tous les professionnels, se heurte à la question politiquement incorrecte du financement. Tous les gouvernements se succèdent et promettent.

2. Que les lois actuelles répondent à l'essentiel des demandes sociales : Cette position est sage et a été soulignée non seulement par les précédentes décisions dudit Conseil, mais également par la Cour européenne des Droits de l'homme. Depuis lors, la sédation profonde et continue, seule nouveauté notable, a montré dans l'accueil réglementé, mesuré et conditionné qui lui a été réservé qu'elle s'inscrivait également dans cette ligne.

3. Que l'assistance au suicide n'est pas traitée par loi de 2016, mais procède de cas marginaux, ne rendant pas souhaitable de modifier le droit existant.

La loi Claeys-Leonetti vient d'être votée. L'assistance au suicide s'est pleinement inscrite dans le débat démocratique présidant à la révision et le débat parlementaire n'était pas en reste. Il ne s'agit donc ni d'oubli, ni de peur, mais d'un choix délibéré du législateur. Le sujet, éminemment délicat, est facilement brandi par certains, usant parfois de ressorts psychologiques un peu trop faciles pour faire réagir le grand public. Ceux qui ont réfléchi posément à la question y ont vu un danger pour les plus fragiles et une démarche non déontologique aux incidences juridiques, sociales et de graves questions de responsabilité.

Qu'il soit permis de parler de la voix des Sages pour le Palais royal. Ces questions ne sont pas à éviter, elles doivent être traitées et, de fait et de droit, l'ont été. Mais le débat doit être posé, réfléchi et prendre en compte tous les éléments : statistiques, humains, médicaux, juridiques...

III 1 Euthanasie et assistance au suicide

\footnotetext{
${ }^{10}$ Comité consultatif national d'éthique, avis 129 : Contribution du. Comité consultatif national d'éthique à la révision de la loi de bioéthique 2018-2019 » (18 septembre 2018).
} 
Aide à mourir, droit à mourir... la question est classique, l'étude terminologique toujours parlante, mais ces questions ne sont pas nouvelles. On saura gré au Conseil d'avoir rappelé ad liminem que la loi Claeys-Leonetti n'a pas deux ans d'existence et qu'elle constituait elle-même une révision de la loi de 2005. Il convient de respecter le travail législatif, ne pas remettre en cause une décision parlementaire qui n'a pas eu le temps de vieillir. L'heure n'est pas même au bilan de la loi, mais seulement à son application.

Sur la question de l'aide à mourir, l'Etude ${ }^{11}$ met en exergue les « risques importants » qui existent, reprenant ici les termes de l'avis 121 du CCNE, euthanasies clandestines et " cas limites ». La France se distingue de quatre pays européens (Pays-Bas, Belgique, Luxembourg et Suisse) en ne voulant pas répondre « aux demandes d'aide à mourir » quand le pronostic vital n'est pas engagé à court terme. C'est éviter de se trouver confronté à des situations telles que celles d'Alda Gross ${ }^{12}$ ou de Jean Mercier ${ }^{13}$, qui ne sont que deux illustrations parmi d'autres des dangers d'une législation éloignée d'une mort imminente. Le Conseil souligne aussi les « fragilités individuelles » et pointe le projecteur sur les risques de sollicitations émanant de personnes atteintes de "troubles psychiques ". Derrière ces troubles, de multiples hypothèses qui renverront entre autres à des personnes dont on pourrait douter de la clairvoyance ou de l'intelligibilité de la demande ou de personnes dépressives.

Franchise et réalisme sont à souligner derrière un paragraphe ${ }^{14}$ qui mérite de n'être pas résumé : « [...] il est difficile d'ignorer l'impact symbolique que l'autorisation d'une aide anticipée à mourir serait susceptible d'avoir sur les personnes les plus vulnérables. En consacrant le droit pour certaines personnes d'obtenir une telle aide, la loi indiquerait, au moins en creux, les contours de ce qui constitue une vie digne. Elle pourrait alors avoir pour conséquence de donner aux personnes susceptibles de répondre aux conditions qu'elle pose et proches des situations qu'elle vise le sentiment imposé de l'indignité ou de l'inutilité de leur vie ».

L'implication du corps médical est une autre question trop souvent maltraitée par les partisans d'un tel droit à mourir. Outre la question essentielle de la déontologie médicale prise à contre-courant, les alternatives de certains pays (Oregon, Suisse...) sont évoquées ${ }^{15}$ qui permettent de dissocier la prescription (par le médecin) des substances létales de l'administration de ces substances (par l'intéressé ou une tiers).

\footnotetext{
${ }^{11}$ Etude citéesupra, $p .126$.

${ }^{12}$ Cour EDH, 14 mai 2013, Alda Gross c/Suisse, Req. $n^{\circ}$ 67810/10 A. Cheynet de Beaupré, La vieille femme et la mort : le soleil vert de la CEDH se lève-t-il sur un droit au suicide assisté ? (RJPF, 2013, $\left.n^{\circ} 9, p .14\right)$; Chr. Byk, JCP G 2013, 1647, et Suicide assisté et obligation positive de l'État, Médecine et Droit 2013, 145 ; N.-J. Mazen et B. Bevière-Boyer, Éthique et droit du vivant, Rev. Gén. de droit médical, 2013.293 ; G. Gonzalez, Quand vous serez bien vieille...: suicide assisté sans raison médicale, JCP G 2013.1115.

Puis : Cour EDH, 30 sept. 2014 : A. Cheynet de Beaupré, Mémoire d'outre-tombe: un abus de droit post mortem! RJPF 2014, no 12, p. 20 ; D. Appanah, À la recherche posthume de l'intention du requérant : l'identification délicate de la requête abusive au sens de la Convention, RTDH, 2015.1053; N. Fricero, Abus du droit de recours individuel, Procédures, 2015.22; L. Burgorgue-Larsen, Requête abusive, AJDA 2015.150.

${ }^{13}$ Cass. Crim., 13 déc. 2017, $n^{\circ} 16-87054$.

${ }^{14}$ Etude p. 126.

${ }^{15}$ Etude p. 127.
} 
III 2 Mal mourir et humilité ${ }^{16}$

Humilité : l'étymologie d'humus (la terre) est particulièrement forte ... Le vocabulaire utilisé est révélateur d'une démarche qui ne veut pas ignorer les difficultés juridiques et humaines sousjacentes et incontournables pour l'un des sujets les plus graves de la Société humaine. A de nombreuses reprises est évoqué l'un des problèmes cruciaux de cette question de la fin de vie : les soins palliatifs. Les appels sont démultipliés, renouvelés, réitérés... on ne sait plus trop comment dire, mais il s'agit toujours (dès avant 2005) de " crier » un insuffisant développement des soins palliatifs, que ce soit dans la création d'unités (dédiées, spécialisées, mobiles...) ou de personnels. Le fond de la question est connu et trivial : I'argent.

${ }^{16}$ Etude p. 128. 
A
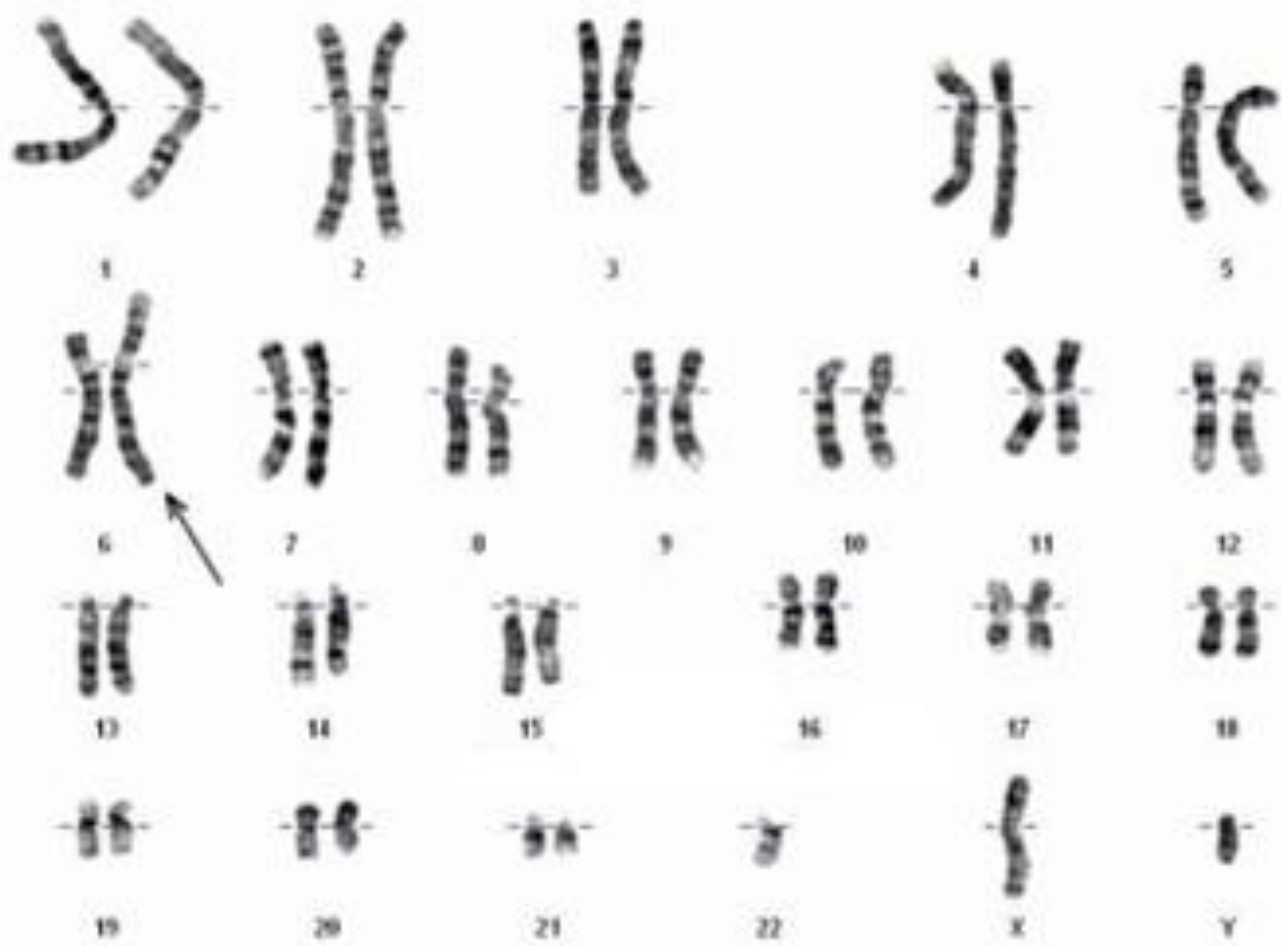

B

C
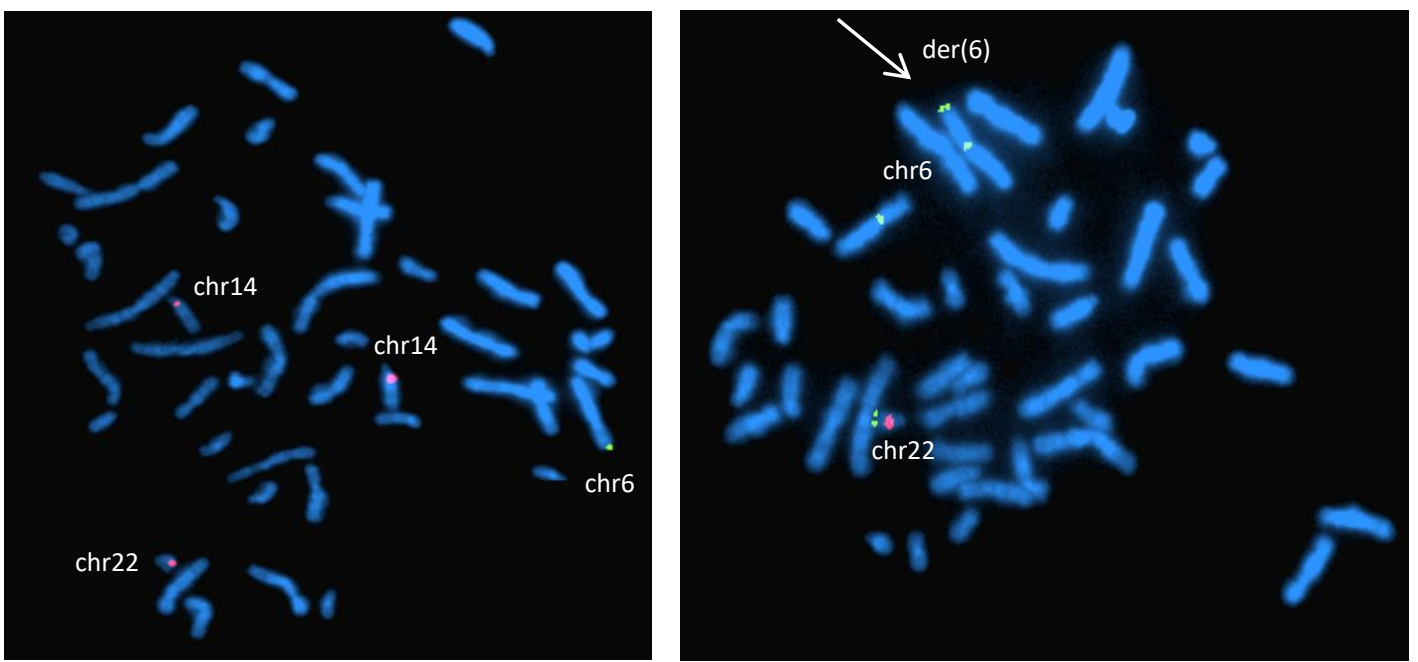

Figure 1 


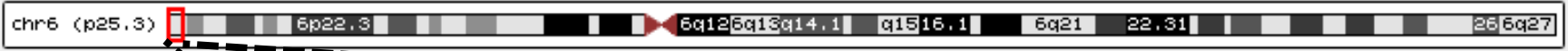

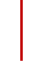

Figure 2 
A

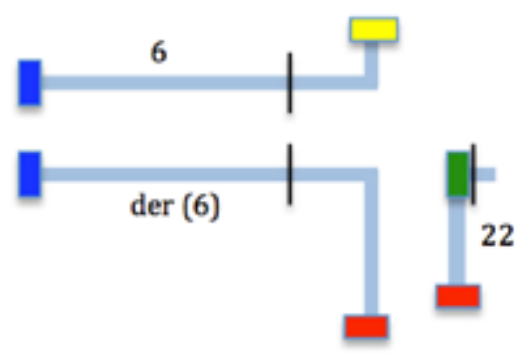

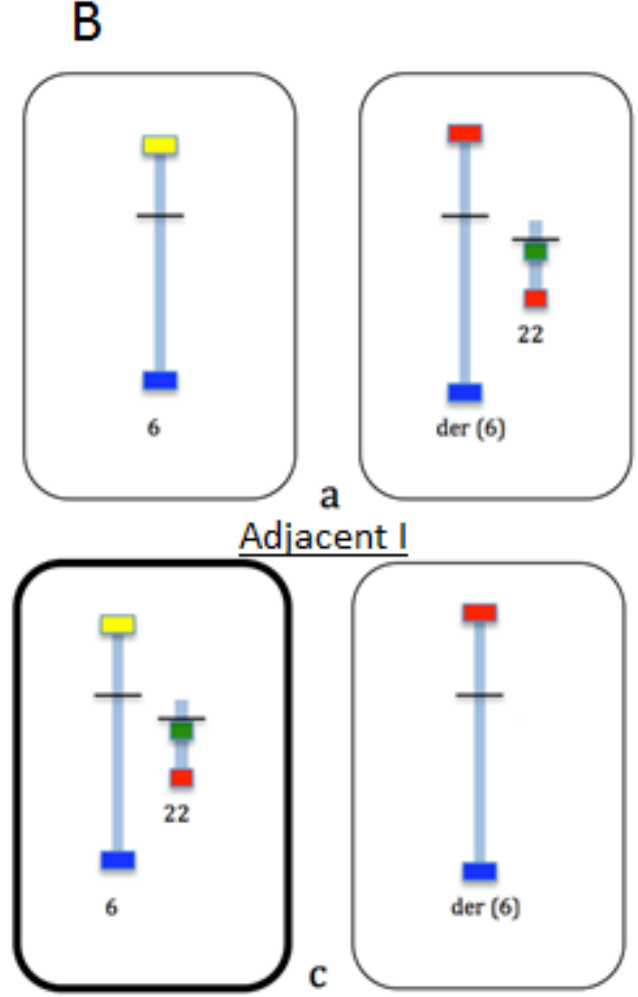

Alternate

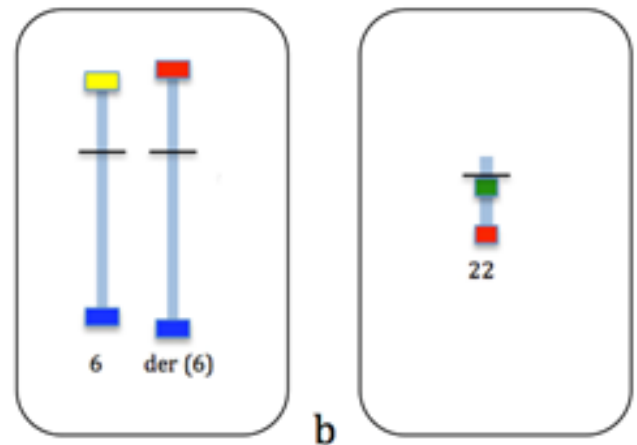

Adjacent II

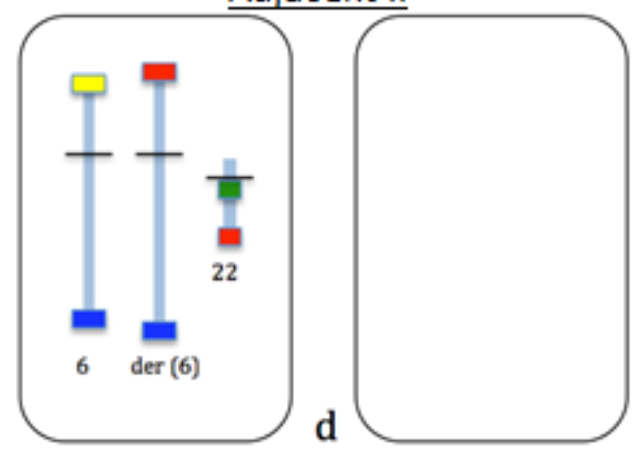

3:1

Figure 3 